\title{
Degree of blood pressure control and the risk of new-onset hyperuricemia in treated hypertensive patients
}

\author{
Qinqin Li ${ }^{1,2}$, Yuanyuan Zhang ${ }^{1}$, Jingjing $\mathrm{Cao}^{2}$, Jingping Zhang ${ }^{2}$, Jing $\mathrm{Nie}^{1}$, Min Liang ${ }^{1}$, Jianping $\mathrm{Li}^{3}$, \\ Yan Zhang ${ }^{3}$, Binyan Wang ${ }^{2}$, Yong Huo ${ }^{3}$, Xiaobin Wang ${ }^{4}$, Fan Fan Hou ${ }^{1}$, Xiping Xu ${ }^{1}$, Xianhui Qin ${ }^{1,2}$ \\ ${ }^{1}$ Division of Nephrology, Nanfang Hospital, Southern Medical University, National Clinical Research Center for Kidney Disease, State Key \\ Laboratory for Organ Failure Research, Guangzhou, China; ${ }^{2}$ Institute of Biomedicine, Anhui Medical University, Hefei, China; ${ }^{3}$ Department \\ of Cardiology, Peking University First Hospital, Beijing, China; ${ }^{4}$ Department of Population, Family and Reproductive Health, Johns Hopkins \\ University Bloomberg School of Public Health, Baltimore, MD, USA \\ Contributions: (I) Conception and design: FF Hou, X Xu, X Qin; (II) Administrative support: FF Hou, X Xu, X Qin; (III) Provision of study materials \\ or patients: FF Hou, X Xu, X Qin; (IV) Collection and assembly of data: J Li, Y Zhang, B Wang, Y Huo, FF Hou, X Qin; (V) Data analysis and \\ interpretation: Q Li, Y Zhang, X Qin; (VI) Manuscript writing: All authors; (VII) Final approval of manuscript: All authors. \\ Correspondence to: Xianhui Qin; Xiping Xu; Fan Fan Hou. Division of Nephrology, Nanfang Hospital, Southern Medical University, National Clinical \\ Research Center for Kidney Disease, State Key Laboratory for Organ Failure Research, Guangzhou 510515, China. Email: pharmaqin@126.com; \\ xipingxu126@126.com; ffhouguangzhou@163.com.
}

Background: The relationship between blood pressure (BP) control and the risk of new-onset hyperuricemia remains uncertain. We aimed to examine the association between degree of time-averaged ontreatment BP control and new-onset hyperuricemia in general hypertensive patients.

Methods: A total of 10,617 hypertensive patients with normal uric acid (UA) concentrations $(<357 \mu \mathrm{mol} / \mathrm{L})$ at baseline were included from the UA Sub-study of the China Stroke Primary Prevention Trial (CSPPT). Participants were randomized to receive a double-blind daily treatment of enalapril $10 \mathrm{mg}$ and folic acid $0.8 \mathrm{mg}$ or enalapril $10 \mathrm{mg}$ alone. BP measurements were taken every three months after randomization. The primary outcome was new-onset hyperuricemia, defined as a UA concentration $\geq 417 \mu \mathrm{mol} / \mathrm{L}$ in men or $\geq 357 \mu \mathrm{mol} / \mathrm{L}$ in women at the exit visit.

Results: Over a median of 4.4 years, 1,664 (15.7\%) participants developed new-onset hyperuricemia. Overall, there was a significantly positive association between time-averaged on-treatment diastolic BP (DBP) and new-onset hyperuricemia (per $10 \mathrm{mmHg}$ increment; OR 1.13; 95\% CI: 1.02-1.26). Consistently, a significantly higher risk of new-onset hyperuricemia was found in participants with time-averaged ontreatment DBP $\geq 82.9$ mmHg (median) (vs. <82.9 mmHg; 17.3\% vs. 14.1\%; OR 1.25; 95\% CI: 1.10-1.44). Furthermore, the lowest new-onset hyperuricemia risk $(12.1 \%)$ was found in those with both time-averaged on-treatment SBP (median: $138.3 \mathrm{mmHg}$ ) and DBP below the median (P-interaction=0.023). The results were similar for time-averaged DBP during the first 12- or 24-month treatment period, or in the analysis using propensity scores. Furthermore, a non-significant higher risk of new-onset hyperuricemia was observed in participants with time-averaged on-treatment SBP $\geq 120 \mathrm{mmHg}(v s .<120 \mathrm{mmHg}$; OR $1.61 ; 95 \%$ CI: 0.88-2.97).

Conclusions: A tight DBP control of $<82.9 \mathrm{mmHg}$ was associated with lower risk of new-onset hyperuricemia among hypertensive patients without hyperuricemia.

Keywords: Diastolic blood pressure control (DBP control); systolic blood pressure control (SBP control); uric acid; new-onset hyperuricemia; hypertension

Submitted Mar 31, 2020. Accepted for publication Aug 28, 2020.

doi: $10.21037 /$ atm-20-3017

View this article at: http://dx.doi.org/10.21037/atm-20-3017 


\section{Introduction}

Hyperuricemia is characterized by an abnormally elevated concentration of serum uric acid (UA) as a direct result of over-production and/or impaired renal excretion of UA in the body $(1,2)$. The prevalence of hyperuricemia varies substantially worldwide, ranging from about $11.4 \%$ to $36 \%(3-5)$. In China, $13.3 \%$ of the population (almost 170 million) has hyperuricemia and the prevalence of hyperuricemia is increasing, partly owing to population aging (6). Hyperuricemia is not only a strong predictor of gout (1), but is also associated with the risk of cancer, chronic kidney disease, stroke, cardiovascular diseases, and mortality (7-9). Furthermore, previous studies have reported that the reduction of UA had significant reno-protective and cardiovascular protective effects (10-12). Therefore, it is of great significance to identify more modifiable risk factors for hyperuricemia and develop effective primary prevention strategies.

Hypertension is a worldwide public health challenge because of its high burden of comorbidity (cardiovascular, kidney disease, etc.) and limited life expectancy $(13,14)$. To date, some cross-sectional studies have shown that hyperuricemia is more common in patients with hypertension than non-hypertension (15-18). However, although a few longitudinal studies have reported that high blood pressure (BP) is associated with the development of hyperuricemia (19-21), the relationship between BP control and the risk of new-onset hyperuricemia in hypertensive adults remains uncertain.

To address this aforementioned gap, our current study, a post-hoc analysis of data from the UA Sub-study of the China Stroke Primary Prevention Trial (CSPPT) (22), aimed to evaluate the association between degree of timeaveraged on-treatment BP control with the risk of newonset hyperuricemia, and to examine any possible effect modifiers among hypertensive patients. We present the article in accordance with the STROBE reporting checklist (available at http://dx.doi.org/10.21037/atm-20-3017).

\section{Methods}

\section{Study design and participants}

The methods and major results of the CSPPT (23-25) and the UA Sub-study of the CSPPT (22) have been described previously. Briefly, the CSPPT was a multicommunity, randomized, double-blind, strictly controlled trial conducted from May 19, 2008 to August 24, 2013 in
32 communities in Jiangsu and Anhui provinces of China. Eligible subjects were men and women aged $45-75$ years with hypertension, defined as seated, resting systolic BP (SBP) $\geq 140 \mathrm{mmHg}$ or diastolic BP (DBP) $\geq 90 \mathrm{mmHg}$ at both the screening and recruitment visit $\mathrm{OR}$ who were taking antihypertensive medication. The major exclusion criteria included history of physician-diagnosed stroke, myocardial infarction (MI), heart failure, post-coronary revascularization, and/or congenital heart disease.

The UA Sub-study $(\mathrm{n}=15,364)$ enrolled CSPPT participants from 20 communities in Jiangsu province, excluding those who were using UA-lowering drugs or with missing UA concentrations at baseline. The current study is a post-hoc analysis of the UA Sub-study.

The parent study (the CSPPT) and the current study were approved by the Ethics Committee of the Institute of Biomedicine, Anhui Medical University, Hefei, China (FWA assurance number: FWA00001263) and our study was conducted in accordance with the Declaration of Helsinki. All participants provided written informed consent.

\section{Intervention and follow-up}

The CSPPT consisted of 3 stages: screening and recruitment, a 3-week run-in treatment period, and a 5-year randomized treatment period. During the run-in period, all eligible participants were given a daily enalapril $10 \mathrm{mg}$ tablet for 3 weeks. In the double-blind treatment period, eligible participants were randomly assigned, in a 1:1 ratio, to a double-blind daily treatment of enalapril $10 \mathrm{mg}$ and folic acid $0.8 \mathrm{mg}$ or enalapril $10 \mathrm{mg}$ alone.

The dosage of the study drugs was fixed during the trial. During the run-in and double-blind treatment period, if BP was not adequately controlled, other classes of anti-hypertensive medications could be prescribed concomitantly, according to a pre-specified algorithm. Step 1: (I) enalapril or enalapril-folic acid tablet daily + nitrendipine (10 mg, bid); or (II) enalapril or enalapril-folic acid tablet daily + hydrochlorothiazide $(25 \mathrm{mg})$ daily; Step 2: enalapril or enalapril-folic acid tablet daily + nitrendipine (10 mg, bid) + hydrochlorothiazide ( $25 \mathrm{mg})$ daily. In Step 1, nitrendipine was the preferred choice. Alpha-blockers, betablockers, angiotensin II receptor blockers (ARBs) and other ACEIs were not recommended. BP control within a normal range (SBP $<140 \mathrm{mmHg}$ and $\mathrm{DBP}<90 \mathrm{mmHg}$ ) was not mandatory.

Participants were followed up every three months. At each follow-up visit, BP was measured; study drug 
compliance, concomitant medication use, adverse events and possible endpoint events were documented by trained research staff and physicians. At the exit visit, final blood samples were collected and assessed.

\section{BP measurements}

After participants had rested for 10 minutes, seated BP was measured by trained research staff using a mercury manometer, following the standard method and with appropriately sized cuffs. Triplicate measurements on the same arm were taken, with at least 2 minutes between readings. The mean SBP and DBP of the three independent measures were used in analysis.

BP measurements were taken at baseline, randomization and every 3 months thereafter. Time-averaged ontreatment SBP or DBP was calculated for each participant using all post baseline results up to the last visit [number of BP measurements during the treatment: median, 16; interquartile range (IQR), 12-18]. In additional sensitivity analyses, we also calculated time-averaged on-treatment SBP or DBP using two alternative methods: (I) using the BP measurements from the randomization visit to the 12 -month visit (number of BP measurements: median, 5; IQR, 4-5); (II) using the measurements from the randomization visit to the 24-month visit (number of BP measurements: median, 8; IQR, 7-9).

\section{Laboratory assays}

Blood samples of all participants were collected at both the baseline and the exit visits. Serum concentrations of UA, total homocysteine (tHcy), lipids, fasting glucose, creatinine, and phosphate were measured using automatic analyzers (Beckman Coulter) at the core laboratory of the National Clinical Research Center for Kidney Disease, Nanfang Hospital, Guangzhou, China. Serum folate was measured by a commercial laboratory with the use of a chemiluminescent immunoassay (New Industrial). The estimated glomerular filtration rate (eGFR) was calculated by the Chronic Kidney Disease Epidemiology Collaboration equation (26).

\section{Outcomes}

The primary outcome was new-onset hyperuricemia in participants with normal UA concentrations $[<357 \mu \mathrm{mol} / \mathrm{L}$ $(6 \mathrm{mg} / \mathrm{dL})]$ at baseline. Hyperuricemia was defined as a UA concentration $\geq 417 \mu \mathrm{mol} / \mathrm{L}(7 \mathrm{mg} / \mathrm{dL})$ in men or $\geq 357 \mu \mathrm{mol} / \mathrm{L}(6 \mathrm{mg} / \mathrm{dL})$ in women $(22,27)$.

The secondary outcome was change in UA concentrations, defined as UA concentrations at the exit visit minus that at baseline.

\section{Statistical analyses}

Population characteristics are presented as mean \pm standard deviation (SD) for continuous variables and proportions for categorical variables. Differences in population characteristics by time-averaged on-treatment SBP or DBP quartiles were compared using ANOVA tests, or chi-square tests, accordingly.

The relationship of time-averaged on-treatment SBP or DBP with new-onset hyperuricemia (primary outcome), and change in serum UA concentrations (secondary outcome) was evaluated using multivariable logistic regression models [odds ratio (OR) and 95\% confidence interval (CI)] and generalized linear regression models, respectively, without and with adjustment for treatment group, age, sex, body mass index (BMI), UA, fasting glucose, total cholesterol (TC), triglycerides (TG), tHcy, folate, eGFR, smoking and drinking status, use of antihypertensive drugs, as well as SBP and DBP at baseline. Furthermore, possible modifications on the association between time-averaged on-treatment DBP (median, <82.9 vs. $\geq 82.9 \mathrm{mmHg}$ ) and new-onset hyperuricemia were also evaluated by stratified analyses and interaction testing.

\section{Propensity scores analysis}

As additional sensitivity analyses, we further evaluated our results in the analysis using propensity score matching method. A non-parsimonious propensity score using variables that might affect BP control or new-onset hyperuricemia was developed to predict the likelihood a participant would be in the different degree of time-averaged on-treatment SBP (median, <138.3 vs. $\geq 138.3 \mathrm{mmHg}$ ) or DBP (median, $<82.9$ vs. $\geq 82.9 \mathrm{mmHg}$ ) control. Participants in the 2 groups with different degree of time-averaged on-treatment BP control were matched 1:1 based on propensity scores. Nearest-neighbor with caliper distance of 0.05 was used for the matching. Standardized differences of post-matched participant characteristics $\leq 10 \%$ between the 2 groups was considered to be balanced.

A two-tailed $\mathrm{P}<0.05$ was considered to be statistically significant in all analyses. Statistical analyses were conducted using R software, version 3.5.0. 


\section{Results}

\section{Characteristics of study participants}

As illustrated in the flow chart (Figure S1), of the 15,364 participants in the UA Sub-study of CSPPT, the final UA measurement at the exit visit was obtained for 13,163 (85.7\%). Participants without UA concentrations at the exit visit did not differ substantially in baseline characteristics from those with UA concentrations (Table S1). Of the 13,163 participants, a total of 10,617 subjects, who did not use UA-lowing drugs during the treatment period, as well as whose baseline UA levels were $<357 \mu \mathrm{mol} / \mathrm{L}$ in the UA Substudy of the CSPPT were included in the final analyses.

The median time-averaged on-treatment SBP or DBP was 138.3 and $82.9 \mathrm{mmHg}$, respectively. Baseline characteristics of the participants by time-averaged ontreatment SBP or DBP quartiles are summarized in Table S2 and Table 1, respectively. Time-averaged on-treatment DBP was positively associated with male sex, BMI, current smoking, current alcohol drinking, SBP, DBP, eGFR, UA and antihypertensive drug usage; and was inversely associated with age, TC, folate, phosphate concentrations, fasting glucose, glucose-lowering drug usage and the prevalence of self-reported diabetes (Table 1). Similar trends were found for time-averaged on-treatment SBP (Table S2).

\section{Time-averaged on-treatment BP and risk of study outcomes}

Over a median follow-up duration of 4.4 years, new-onset hyperuricemia occurred in 1,664 (15.7\%) patients.

The association between time-averaged on-treatment DBP and new-onset hyperuricemia is presented in Figure $1 \mathrm{~A}$. Overall, there was a significant positive association between time-averaged on-treatment DBP and the risk of new-onset hyperuricemia (per $10 \mathrm{mmHg}$ increment; OR 1.13; 95\% CI: 1.02-1.26) (Table 2). When time-averaged on-treatment DBP was assessed as quartiles, a significantly higher risk of new-onset hyperuricemia was found in participants with time-averaged on-treatment DBP $\geq 82.9 \mathrm{mmHg}$ (median) (vs. $<82.9 \mathrm{mmHg} ; 17.3 \%$ vs. 14.1\%; OR 1.25; 95\% CI: 1.10-1.44) (Table 2). Similar results were found for change in serum UA concentrations (secondary outcome) (Figure 1B, Table S3) and the exit UA concentrations (Figure S2).

Consistently, when modeled as clinical categories, compared with participants with time-averaged ontreatment DBP $<85 \mathrm{mmHg}$, the adjusted odds ratios $(95 \%$
CI) for participants with time-averaged DBP 85 to $<90$, and $\geq 90 \mathrm{mmHg}$ were 1.16 (95\% CI: $0.99-1.34)$ and 1.33 (95\% CI: 1.11-1.59) (P for trend =0.001), respectively. Accordingly, a significantly higher risk of new-onset hyperuricemia was observed in participants with timeaveraged DBP $\geq 85$ mmHg (OR 1.21; 95\% CI: 1.06-1.39), compared with those with time-averaged DBP $<85 \mathrm{mmHg}$ (Table S4).

In addition, there were significant differences in the use of calcium channel blockers, diuretics, and glucoselowering drugs during the treatment period among those with different time-averaged on-treatment DBP levels (Table S5). However, further adjustment for concomitant use of calcium channel blockers, diuretics and glucoselowering drugs during the treatment period did not substantially change the results (Table S6). Moreover, further adjustment for treatment (enalapril or enalapril-folic acid) compliance (Table S7), change in eGFR (Table S8), or change in BMI (Table S9) during the treatment period also did not essentially affect the results. The similar results were also observed when UA concentrations $<417 \mu \mathrm{mol} / \mathrm{L}$ for male and $<357 \mu \mathrm{mol} / \mathrm{L}$ for female at baseline were included the analysis (Table S10).

Overall, there was no significant association between time-averaged on-treatment SBP and the primary or secondary outcomes (Table 2, Table S3, Figure S3). Nevertheless, compared with those with time-averaged ontreatment SBP $<120 \mathrm{mmHg}$, a non-significant higher risk of new-onset hyperuricemia was observed in participants with time-averaged on-treatment $\mathrm{SBP} \geq 120 \mathrm{mmHg}$ (OR 1.61; 95\% CI: 0.88-2.97) (Table S11).

\section{Time-averaged BP during the first 12-month or 24-month treatment period and subsequent new-onset hyperuricemia}

When time-averaged DBP during the first 12-month treatment period was assessed as quartiles, the adjusted ORs (95\% CI) for participants in the third (84.5 to $<89.7 \mathrm{mmHg}$ ) and fourth quartiles $(\geq 89.7 \mathrm{mmHg})$ were $1.17(0.97-1.40)$ and $1.27(1.04-1.55)$, respectively, compared with participants in quartile $1(<79.6 \mathrm{mmHg}$; $\mathrm{P}$ for trend $=0.024)$ (Table S12).

When time-averaged DBP during the first 24-month treatment period was assessed as quartiles, the adjusted ORs (95\% CI) for participants in the third (83.8 to $<88.6 \mathrm{mmHg}$ ) and fourth quartiles $(\geq 88.6 \mathrm{mmHg}$ ) were 1.26 (1.05-1.51) and $1.29(1.05-1.58)$, respectively, compared with participants in quartile $1(<79.0 \mathrm{mmHg} ; \mathrm{P}$ for trend 
Table 1 Characteristics of study participants according to quartiles of time-averaged on-treatment DBP

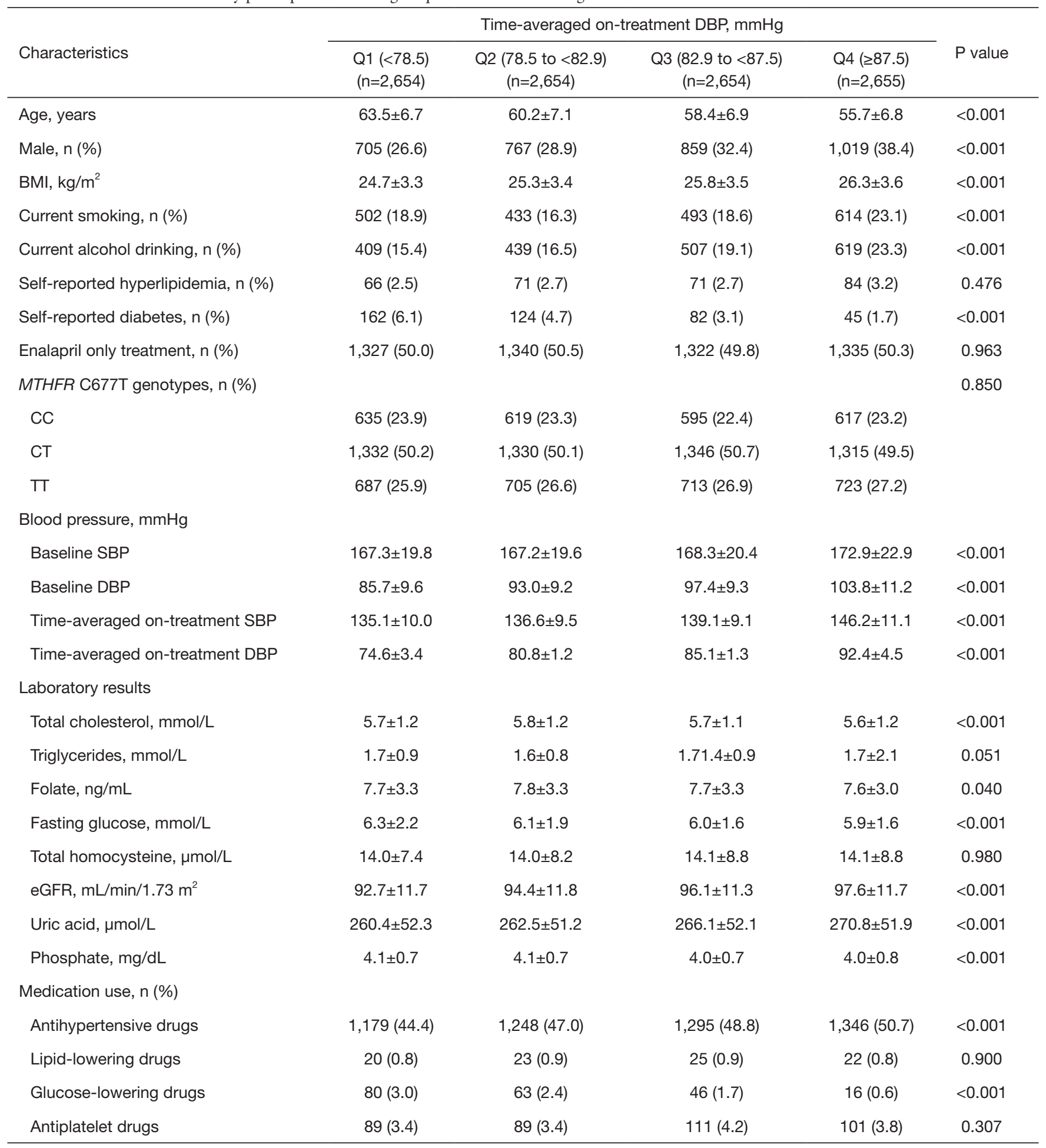

Variables are presented as mean \pm SD or $n(\%)$. BMI, body mass index; DBP, diastolic blood pressure; eGFR, estimated glomerular filtration rate; MTHFR, 5, 10-methylenetetrahydrofolate reductase; SBP, systolic blood pressure. 

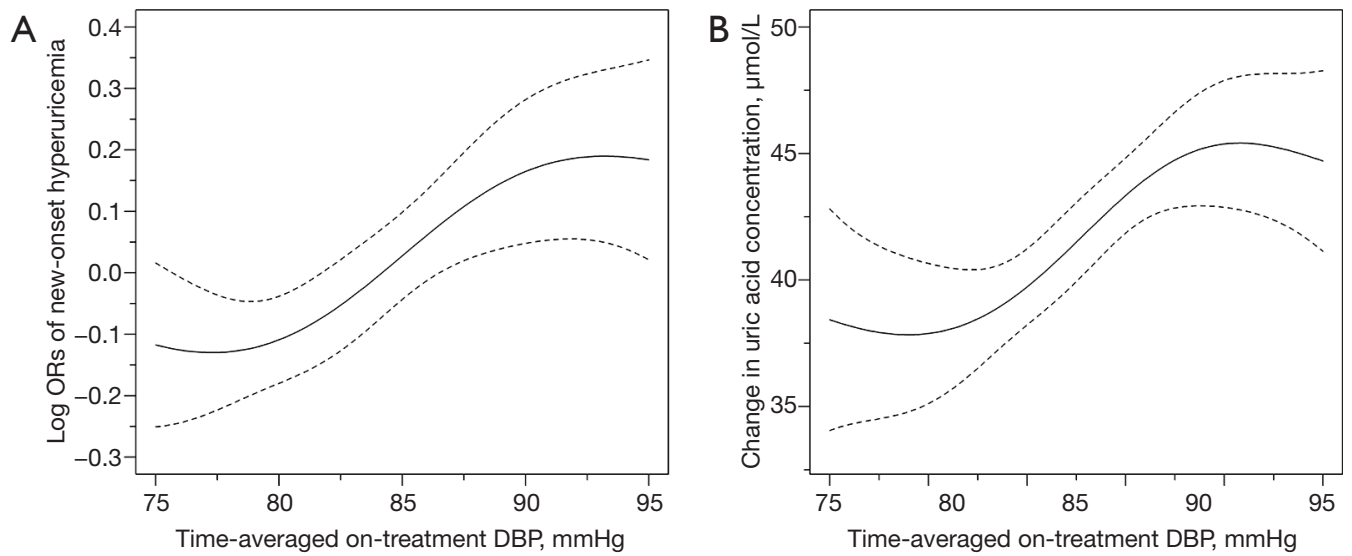

Figure 1 Relationship of time-averaged on-treatment diastolic blood pressure (DBP) with new-onset hyperuricemia (A) and change in uric acid concentrations (B)*. *, adjusted for treatment group, age, sex, BMI, UA, fasting glucose, total cholesterol, TG, tHcy, folate, eGFR, smoking and drinking status, use of antihypertensive drugs, and SBP and DBP at baseline.

Table 2 Association between time-averaged on-treatment blood pressure and new-onset hyperuricemia

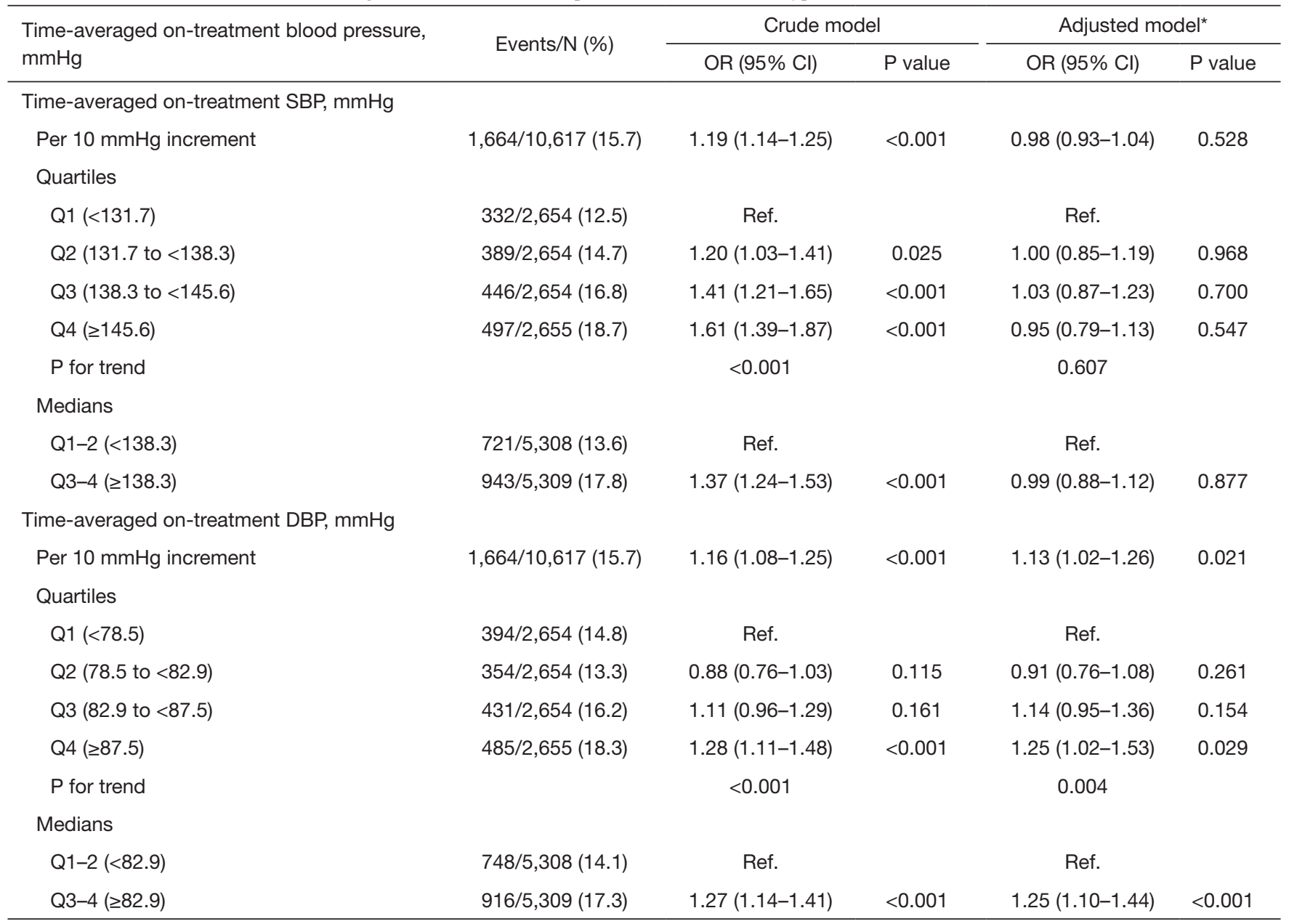

*, adjusted for treatment group, age, sex, BMI, UA, fasting glucose, total cholesterol, TG, tHcy, folate, eGFR, smoking and drinking status, use of antihypertensive drugs, and SBP and DBP at baseline. 


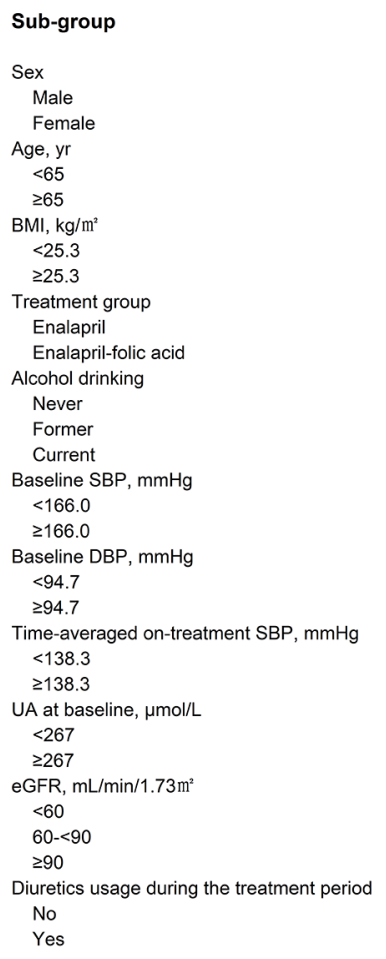

\begin{tabular}{|c|c|}
\hline Size & $\begin{array}{c}<82.9 \mathrm{mmHg} \\
\text { Events(\%) }\end{array}$ \\
\hline 3350 & $153(10.4)$ \\
\hline 7267 & $595(15.5)$ \\
\hline 7948 & $406(12.0)$ \\
\hline 2669 & $342(17.8)$ \\
\hline 5306 & $329(11.0)$ \\
\hline 5309 & $419(18.0)$ \\
\hline 5324 & $390(14.6)$ \\
\hline 5293 & $358(13.6)$ \\
\hline 3872 & $608(14.5)$ \\
\hline 306 & $29(10.4)$ \\
\hline 1126 & $157(13.9)$ \\
\hline 5267 & $311(11.2)$ \\
\hline 5350 & $437(17.3)$ \\
\hline 5228 & $503(13.5)$ \\
\hline 5389 & $245(15.4)$ \\
\hline 5308 & $400(12.1)$ \\
\hline 5309 & $348(17.4)$ \\
\hline 5255 & $203(7.3)$ \\
\hline 5362 & $545(21.6)$ \\
\hline 120 & $24(31.2)$ \\
\hline 2679 & $315(20.3)$ \\
\hline 7660 & $402(11.2)$ \\
\hline 4219 & $284(11.3)$ \\
\hline 6398 & 464 (16.5) \\
\hline
\end{tabular}

$$
\begin{gathered}
\geq 82.9 \mathrm{mmHg} \\
\text { Events(\%) } \\
230(12.2) \\
686(20.0) \\
751(16.5) \\
165(22.0) \\
275(11.8) \\
641(21.5) \\
479(18.0) \\
437(16.5) \\
718(18.5) \\
40(13.1) \\
157(13.9) \\
352(14.2) \\
564(20.0) \\
229(15.2)) \\
687(18.1) \\
321(16.1) \\
595(18.0) \\
205(8.3) \\
711(25.0) \\
17(39.5) \\
261(23.1) \\
628(15.5) \\
217(12.7) \\
699(19.4) \\
\end{gathered}
$$

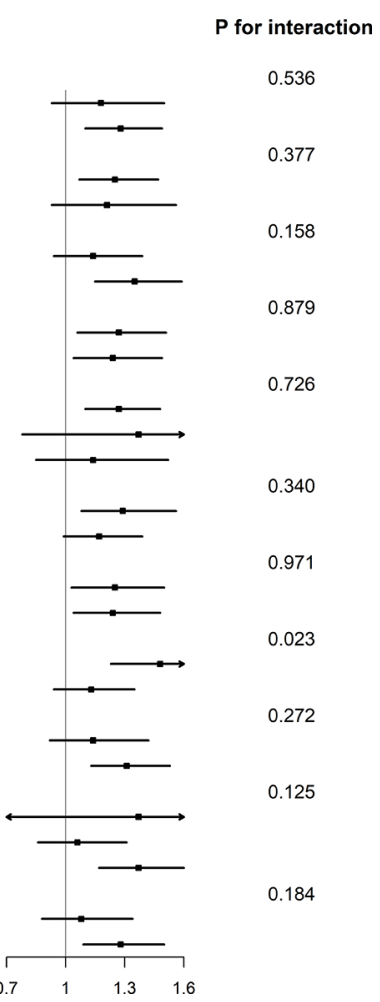

Figure 2 Stratified analyses of the association between time-averaged on-treatment DBP (median, $<82.9 v s . \geq 82.9 \mathrm{mmHg}$ ) and new-onset hyperuricemia*. *, adjusted, if not stratified, for treatment group, age, sex, BMI, UA, fasting glucose, total cholesterol, TG, tHcy, folate, eGFR, smoking and drinking status, use of antihypertensive drugs, and SBP and DBP at baseline.

$=0.001)($ Table S13).

\section{Stratified analyses by potential effect modifiers}

A stronger, positive association was observed between time-averaged on-treatment DBP (median, <82.9 vs. $\geq 82.9 \mathrm{mmHg}$ ) and new-onset hyperuricemia among participants with lower time-averaged on-treatment SBP (median: <138.3; OR 1.48; 95\% CI: 1.23-1.78; vs. $\geq 138.3 \mathrm{mmHg}$; OR 1.13; 95\% CI: 0.94-1.35; $P$-interaction $=0.023$ ) (Figure 2). That is to say, the lowest new-onset hyperuricemia risk $(12.1 \%)$ was found in those with both lower DBP and lower SBP levels.

None of the other variables, including sex, age $(<65$ $v s . \geq 65$ years), treatment group (enalapril $v s$. enalaprilfolic acid), BMI (median: $<25.3 v s . \geq 25.3 \mathrm{~kg} / \mathrm{m}^{2}$ ), SBP (median: <166.0 vs. $\geq 166.0 \mathrm{mmHg}$ ), DBP (median: <94.7 vs. $\geq 94.7 \mathrm{mmHg}$ ), serum UA concentrations (median: $<267 v s . \geq 267 \mu \mathrm{mol} / \mathrm{L})$, eGFR (<60 vs. 60 to $<90 v s$. $\geq 90 \mathrm{~mL} / \mathrm{min} / 1.73 \mathrm{~m}^{2}$ ), smoking (never $v s$. former $v s$. current), drinking (never $v s$. former $v s$. current), TC (median: $<5.6 v s . \geq 5.6 \mathrm{mmol} / \mathrm{L})$, tHcy $(<15 v s . \geq 15 \mu \mathrm{mol} / \mathrm{L}$ ), diabetes (no vs. yes) and phosphate concentrations (median: $<4.0 v s . \geq 4.0 \mathrm{mg} / \mathrm{dL}$ ) at baseline, as well as diuretics usage during the treatment period (no vs. yes) significantly modified the association between time-averaged ontreatment DBP (median, $<82.9 v s . \geq 82.9 \mathrm{mmHg}$ ) and the risk of new-onset hyperuricemia (All P-interactions $>0.05$ ) (Figure 2, Figure S4).

\section{Propensity scores analysis}

After propensity score matching, 6,670 participants (3,335 in each group) were included in the analysis for SBP \& new-onset hyperuricemia association, and 5,346 participants (2,673 in each group) were included in the analysis for DBP \& new-onset hyperuricemia association. Candidate variables used in the development of the propensity score have been listed in Table S14. All the post-matched participant characteristics were highly balanced (Table S14, Figures S5,S6). Consistently, a significantly higher risk of newonset hyperuricemia was found in participants with time- 
Table 3 Association between time-averaged on-treatment blood pressure and new-onset hyperuricemia in the propensity scores matching analysis

\begin{tabular}{|c|c|c|c|}
\hline $\begin{array}{l}\text { Time-averaged on-treatment } \\
\text { blood pressure, } \mathrm{mmHg}\end{array}$ & Events/N (\%) & OR $(95 \% \mathrm{Cl})$ & $P$ \\
\hline \multicolumn{4}{|c|}{ Time-averaged on-treatment SBP, $\mathrm{mmHg}$} \\
\hline Q1-2 (<138.3) & $539 / 3,335(16.2)$ & Ref. & \\
\hline Q3-4 ( $\geq 138.3)$ & 497/3,335 (14.9) & $0.91(0.80-1.04)$ & 0.156 \\
\hline \multicolumn{4}{|c|}{ Time-averaged on-treatment DBP, $\mathrm{mmHg}$} \\
\hline Q1-2 (<82.9) & $364 / 2,673(13.6)$ & Ref. & \\
\hline Q3-4 ( $\geq 82.9)$ & 430/2,673 (16.1) & $1.20(1.04-1.40)$ & 0.016 \\
\hline
\end{tabular}

DBP, diastolic blood pressure; SBP, systolic blood pressure.

averaged on-treatment DBP $\geq 82.9 \mathrm{mmHg}$ (median) (vs. $<82.9 \mathrm{mmHg} ; 16.1 \%$ vs. $13.6 \%$; OR 1.20 ; $95 \%$ CI: $1.04-$ 1.40). However, there was no significant relation of timeaveraged on-treatment SBP with new-onset hyperuricemia (Table 3).

\section{Discussion}

Our current study demonstrated that a tight DBP control of $<82.9 \mathrm{mmHg}$ was associated with a lower risk of new-onset hyperuricemia among treated hypertensive patients without hyperuricemia, especially in those with lower time-averaged on-treatment SBP during the treatment period.

A few prospective studies (19-21) have assessed the association between BP and the risk of hyperuricemia. Ryu et al. (19) found that participants with hypertension had a higher incidence of hyperuricemia in middle-aged South Korean men. Consistently, Nakanishi et al. (21) reported that high mean $\mathrm{BP}$ was a contributory factor in the development of hyperuricemia in hyperuricemia-free Japanese men. Kuwabara et al. (20) also demonstrated that hypertension was one of the risk factors for developing hyperuricemia in Japanese adults. However, few studies have been conducted to evaluate the association between $\mathrm{BP}$ control and the risk of hyperuricemia. The current report was motivated by the limited data available regarding the relationship between $\mathrm{BP}$ control and new-onset hyperuricemia in general hypertensive patients, and was further prompted by the opportunity to utilize data from a large, randomized controlled trial on patients with regular antihypertensive treatment and BP measurements to address this knowledge gap.
Our study first found that a better DBP control was associated with a lower risk of new-onset hyperuricemia among hypertensive patients, independent of multiple confounding factors including BMI, alcohol drinking, eGFR, BP, UA at baseline, as well as diuretic usage, and change in eGFR and BMI during the treatment period. While biological mechanisms underlying our observed BP control-new onset hyperuricemia association remain to be determined, our findings are biologically plausible. First, hypertension may result in renal vascular resistance accompanied by reduction in renal blood flow (28). As renal blood flow decreases, an increase in proximal sodium and UA absorption occurs, which may contribute to increasing serum UA levels $(19,29,30)$. Consistently, it has been reported that the administration of angiotensin II reduces renal urate clearance in combination with a significant decrease in renal blood flow (2,28,31-33). Furthermore, Messerli et al. (18) reported an inverse association between renal blood flow and UA levels, and a positive relation of renal vascular resistance with UA concentrations in a study on general hypertensive patients. Second, microvascular damage associated with hypertension may result in local tissue ischemia (29). In ischemic conditions, adenosine triphosphate is degraded to adenine and xanthine, and the conversion of xanthine dehydrogenase to xanthine oxidase is simultaneously increased, which leads to increased UA production (2,30,33-35). Overall, renal microvascular damage and intrarenal ischemia may contribute to the major explanation for the high, new-onset hyperuricemia burden in hypertensive patients. We speculate that BP control may decrease microvascular resistance or damage, improve renal blood flow and tissue ischemia, and therefore, reduce the risk of new-onset hyperuricemia. Of note, Figure 1 showed that the relation of time-averaged DBP with newonset hyperuricemia seemed to be "S" shaped. That is to say, the beneficial effect of BP reduction on new-onset hyperuricemia may reach a plateau in those with relatively optimal BP levels. On the other hand, we speculated that when there has already been a relatively serious renal microvascular damage associated with high $\mathrm{BP}$, the impaired renal blood flow and intrarenal ischemia could not continuously increase with the further increment of BP, and, therefore, there may be a plateau, but not a continuously increased new-onset hyperuricemia risk. Future studies are warrened to further examine the findings and the underlying mechanisms of BP control on the risk of hyperuricemia.

However, our study found no significant association between time-averaged on-treatment SBP during the 
treatment period and hyperuricemia risk. The possible explanations included, first, elevated SBP is mainly associated with large artery stiffness, while DBP rises with increases in peripheral vascular resistance (36). As such, at a given sample size, DBP control may possibly have a greater effect on renal blood flow and change in UA levels, and therefore we mainly observed the significant association between DBP control and new-onset hyperuricemia in our current study. Second, we really found a non-significant increased risk of new-onset hyperuricemia in participants with time-averaged on-treatment $\mathrm{SBP} \geq 120 \mathrm{mmHg}$ ( $v s$. $<120 \mathrm{mmHg}$; OR 1.61; 95\% CI: 0.88-2.97). Because only few participants $(1.7 \%)$ reached a time-averaged ontreatment SBP goal of $<120 \mathrm{mmHg}$, these results may just indicate that our current study was underpowered for evaluating the relation of time-averaged SBP $<120 \mathrm{mmHg}$ (vs. $\geq 120 \mathrm{mmHg}$ ) with new-onset hyperuricemia. Of note, in the stratified analysis (Figure 2), the lowest new-onset hyperuricemia risk was found in those with both lower DBP and lower SBP levels. These results suggested that a relatively stricter both SBP and DBP control may lead to a greater reduction of new-onset hyperuricemia in general hypertensive patients. However, more studies are needed to verify our results and hypothesis.

Some limitations of the current study should be noted. First, this was a post-hoc analysis of the UA Sub-study of the CSPPT. Although a broad array of covariates has been adjusted for in the regression models, we cannot exclude residual confounding from unmeasured risk factors. Second, serum UA concentrations were only assessed at the baseline and exit visits. More frequent measurements of serum UA would have allowed for a more accurate assessment of the relation of $\mathrm{BP}$ control and new-onset hyperuricemia. Third, in the CSPPT, all participants used enalapril during the treatment period. The generalizability of our results to hypertensive adults without the use of angiotensinconverting enzyme inhibitors remains to be determined. Fourth, although the positive relation of UA levels with risk of stroke and mortality in previous studies (8), our current study did not find the significant association between baseline UA and first stroke (per SD increment; HR, 0.98, 95\% CI: 0.88-1.09) (Figure S7A). However, our study found a positive association between baseline UA and all-cause mortality (per SD increment; HR, 1.15, 95\% CI: 1.03-1.29) (Figure S7B). Therefore, the association between UA levels and cardiovascular diseases in treated hypertensive patients still should be further investigated in future studies. Fifth, our study mainly suggested that a tight BP control was associated with lower risk of new-onset hyperuricemia. However, our study was underpowered to detect the optimal BP levels for the new-onset hyperuricemia. Due to these limitations, our results should be regarded as hypothesis generating. Confirmation of our findings in a large-scale clinical trial of participants who are randomized to different on-treatment SBP targets is essential.

\section{Conclusions}

In conclusion, there was a positive association between DBP control and the risk of new-onset hyperuricemia in treated hypertensive adults. If further confirmed, our data provide some evidence for the adoption of stricter BP goals in general hypertensive patients for preventing hyperuricemia. These findings could have important implications for clinical practice and guidelines.

\section{Acknowledgments}

Funding: The study was supported by funding from the following: the National Natural Science Foundation of China (81973133,81730019); the National Key Research and Development Program (2016YFE0205400, 2018ZX09739010, 2018ZX09301034003); the Science and Technology Planning Project of Guangzhou, China (201707020010); the Science, Technology and Innovation Committee of Shenzhen (JSGG20170412155639040, GJHS20170314114526143, JSGG20180703 155802047); the Economic, Trade and Information Commission of Shenzhen Municipality (20170505161556110, 20170505160926390); the Research Fund Program of Guangdong Provincial Key Laboratory of Renal Failure Research; and Clinical Innovation Research Program of Guangzhou Regenerative Medicine and Health Guangdong Laboratory (2018ZR0201003).

\section{Footnote}

Reporting Checklist: The authors have completed the STROBE reporting checklist. Available at http://dx.doi. org/10.21037/atm-20-3017

Data Sharing Statement: Available at http://dx.doi. org/10.21037/atm-20-3017

Peer Review File: Available at http://dx.doi.org/10.21037/ atm-20-3017 
Conflicts of Interest: All authors have completed the ICMJE uniform disclosure form (available at http://dx.doi. org/10.21037/atm-20-3017). FFH reports grants from the Research Fund Program of Guangdong Provincial Key Laboratory of Renal Failure Research, grants from Clinical Innovation Research Program of Guangzhou Regenerative Medicine and Health Guangdong Laboratory, during the conduct of the study. XX reports grants from National Key Research and Development Program, grants from Science and Technology Planning Project of Guangzhou, China, grants from Science, Technology and Innovation Committee of Shenzhen, grants from Economic, Trade and Information Commission of Shenzhen Municipality, during the conduct of the study. XQ reports grants from National Natural Science Foundation of China, during the conduct of the study. The other authors have no conflicts of interest to declare.

Ethical Statement: The authors are accountable for all aspects of the work in ensuring that questions related to the accuracy or integrity of any part of the work are appropriately investigated and resolved. The study was approved by the Ethics Committee of the Institute of Biomedicine, Anhui Medical University, Hefei, China (FWA assurance number: FWA00001263) and our study was conducted in accordance with the Declaration of Helsinki. All participants provided written informed consent.

Open Access Statement: This is an Open Access article distributed in accordance with the Creative Commons Attribution-NonCommercial-NoDerivs 4.0 International License (CC BY-NC-ND 4.0), which permits the noncommercial replication and distribution of the article with the strict proviso that no changes or edits are made and the original work is properly cited (including links to both the formal publication through the relevant DOI and the license). See: https://creativecommons.org/licenses/by-nc-nd/4.0/.

\section{References}

1. Neogi T. Clinical practice. Gout. N Engl J Med 2011;364:443-52.

2. Maiuolo J, Oppedisano F, Gratteri S, et al. Regulation of uric acid metabolism and excretion. Int J Cardiol 2016;213:8-14.

3. Chen-Xu M, Yokose C, Rai SK, et al. Contemporary Prevalence of Gout and Hyperuricemia in the United States and Decadal Trends: The National Health and
Nutrition Examination Survey, 2007-2016. Arthritis Rheumatol 2019;71:991-9.

4. Kim Y, Kang J, Kim G. Prevalence of hyperuricemia and its associated factors in the general Korean population: an analysis of a population-based nationally representative sample. Clin Rheumatol 2018;37:2529-38.

5. Lee MS, Lin SC, Chang HY, et al. High prevalence of hyperuricemia in elderly Taiwanese. Asia Pac J Clin Nutr 2005;14:285-92.

6. Liu R, Han C, Wu D, et al. Prevalence of Hyperuricemia and Gout in Mainland China from 2000 to 2014: A Systematic Review and Meta-Analysis. Biomed Res Int 2015;2015:762820.

7. Li L, Yang C, Zhao Y, et al. Is hyperuricemia an independent risk factor for new-onset chronic kidney disease? A systematic review and meta-analysis based on observational cohort studies. BMC Nephrol 2014;15:122.

8. Chen JH, Chuang SY, Chen HJ, et al. Serum uric acid level as an independent risk factor for all-cause, cardiovascular, and ischemic stroke mortality: A Chinese cohort study. Arthritis Rheum 2009;61:225-32.

9. Yang J, Wang Y, Zhao Q, et al. Association of serum uric acid with increased risk of cancer among hypertensive Chinese. Int J Cancer 2017;141:112-20.

10. Su X, Xu B, Yan B, et al. Effects of uric acid-lowering therapy in patients with chronic kidney disease: A metaanalysis. PLoS One 2017;12:e0187550.

11. Smink PA, Bakker SJL, Laverman GD, et al. An initial reduction in serum uric acid during angiotensin receptor blocker treatment is associated with cardiovascular protection. J Hypertens 2012;30:1022-8.

12. Miao Y, Ottenbros SA, Laverman GD, et al. Effect of a Reduction in Uric Acid on Renal Outcomes During Losartan Treatment. Hypertension 2011;58:2-7.

13. Whelton PK, Carey RM, Aronow WS, et al. 2017 ACC/ AHA/AAPA/ABC/ ACPM/AGS/APhA/ASH/ASPC/ NMA/PCNA Guideline for the Prevention, Detection, Evaluation, and Management of High Blood Pressure in Adults. J Am Coll Cardiol 2018;71:e127-248.

14. Li Y, Liang M, Jiang C, et al. Impact of achieved blood pressure on renal function decline and first stroke in hypertensive patients with chronic kidney disease. Nephrol Dial Transplant 2018;33:409-17.

15. Redon P, Maloberti A, Facchetti R, et al. Gender-related differences in serum uric acid in treated hypertensive patients from central and east European countries. J Hypertens 2019;37:380-8.

16. Ramsay LE. Hyperuricaemia in hypertension: role of 
alcohol. BMJ 1979;1:653-54.

17. Cannon PJ, Stason WB, Demartini FE, et al. Hyperuricemia in primary and renal hypertension. N Engl J Med 1966;275:457-64.

18. Messerli FH, Frohlich ED, Dreslinski GR, et al. Serum uric acid in essential hypertension: an indicator of renal vascular involvement. Ann Intern Med 1980;93:817-21.

19. Ryu S, Chang Y, Zhang Y, et al. A Cohort Study of Hyperuricemia in Middle-aged South Korean Men. Am J Epidemiol 2012;175:133-43.

20. Kuwabara M, Borghi C, Cicero AFG, et al. Elevated serum uric acid increases risks for developing high LDL cholesterol and hypertriglyceridemia: A five-year cohort study in Japan. Int J Cardiol 2018;261:183-8.

21. Nakanishi N, Tatara K, Nakamura K, et al. Risk factors for the incidence of hyperuricaemia: a 6-year longitudinal study of middle-aged Japanese men. Int J Epidemiol 1999;28:888-93.

22. Qin X, Li Y, He M, et al. Folic acid therapy reduces serum uric acid in hypertensive patients: a substudy of the China Stroke Primary Prevention Trial (CSPPT). Am J Clin Nutr 2017;105:882-9.

23. Huo Y, Li J, Qin X, et al. Efficacy of folic acid therapy in primary prevention of stroke among adults with hypertension in China: the CSPPT randomized clinical trial. JAMA 2015;313:1325-35.

24. Qin X, Li J, Zhang Y, et al. Effect of folic acid supplementation on risk of new-onset diabetes in adults with hypertension in China: Findings from the China Stroke Primary Prevention Trial (CSPPT). J Diabetes 2016;8:286-94.

25. Qin X, Spence JD, Li J, et al. Interaction of serum vitamin B12 and folate with MTHFR genotypes on risk of ischemic stroke. Neurology 2020;94: e1126-36.

26. Levey AS, Stevens LA, Schmid CH, et al. A New Equation to Estimate Glomerular Filtration Rate. Ann Intern Med

Cite this article as: Li Q, Zhang Y, Cao J, Zhang J, Nie J, Liang M, Li J, Zhang Y, Wang B, Huo Y, Wang X, Hou FF, $\mathrm{Xu} \mathrm{X}$, Qin X. Degree of blood pressure control and the risk of new-onset hyperuricemia in treated hypertensive patients. Ann Transl Med 2020;8(21):1434. doi: 10.21037/atm-20-3017
2009;150:604-12.

27. Zhang W, Doherty M, Pascual E, et al. EULAR evidencebased recommendations for gout. Part I: Diagnosis. Report of a task force of the Standing Committee for International Clinical Studies Including Therapeutics (ESCISIT). Ann Rheum Dis 2006;65:1301-11.

28. Ponnuchamy B, Khalil RA. Cellular mediators of renal vascular dysfunction in hypertension. Am J Physiol Regul Integr Comp Physiol 2009;296:R1001-18.

29. Johnson RJ, Kivlighn SD, Kim YG, et al. Reappraisal of the pathogenesis and consequences of hyperuricemia in hypertension, cardiovascular disease, and renal disease. Am J Kidney Dis 1999;33:225-34.

30. Mazzali M, Kanbay M, Segal MS, et al. Uric Acid and Hypertension: Cause or Effect? Curr Rheumatol Rep 2010;12:108-17.

31. Ferris TF, Gorden P. Effect of angiotensin and norepinephrine upon urate clearance in man. Am J Med 1968;44:359-65.

32. Polichnowski AJ, Griffin KA, Long J, et al. Blood pressurerenal blood flow relationships in conscious angiotensin II- and phenylephrine-infused rats. Am J Physiol Renal Physiol 2013;305:F1074-84.

33. Mennuni S, Rubattu S, Pierelli G, et al. Hypertension and kidneys: unraveling complex molecular mechanisms underlying hypertensive renal damage. J Hum Hypertens 2014;28:74-9.

34. Engerson TD, McKelvey TG, Rhyne DB, et al. Conversion of xanthine dehydrogenase to oxidase in ischemic rat tissues. J Clin Invest 1987;79:1564-70.

35. Strazzullo P, Puig JG. Uric acid and oxidative stress: Relative impact on cardiovascular risk. Nutr Metab Cardiovasc Dis 2007;17:409-14.

36. Franklin SS, Pio JR, Wong ND, et al. Predictors of NewOnset Diastolic and Systolic Hypertension. Circulation 2005;111:1121-7. 